\title{
Gut Microbiome and Brain Functional Connectivity in Infants- A Preliminary Study Focusing on the Amygdala
}

\author{
Wei Gao ${ }^{1,2}$, Andrew P. Salzwedel ${ }^{1}$, Alexander L. Carlson ${ }^{3}$, Kai Xia ${ }^{4}$, M. Andrea Azcarate- \\ Peril $^{5,6}$, Martin A. Styner ${ }^{4,7}$, Amanda L. Thompson ${ }^{8,9}$, Xiujuan Geng ${ }^{10}$, Barbara D. \\ Goldman $^{11,12}$, John H. Gilmore ${ }^{4}$, and Rebecca C. Knickmeyer ${ }^{4,13,14}$ \\ 1.Cedars-Sinai Medical Center. Department of Biomedical Sciences and Imaging, Biomedical \\ Imaging Research Institute. Los Angeles, California. \\ 2.University of California, Department of Medicine. Los Angeles, California. \\ 3.University of North Carolina at Chapel Hill, Neuroscience Curriculum. Chapel Hill, NC. \\ 4. University of North Carolina at Chapel Hill, Department of Psychiatry. Chapel Hill, NC. \\ 5. University of North Carolina at Chapel Hill, Department of Medicine. Chapel Hill, NC. \\ 6. University of North Carolina at Chapel Hill, Microbiome Core Facility. Chapel Hill, NC. \\ 7. University of North Carolina at Chapel Hill, Department of Computer Science. Chapel Hill, NC. \\ 8. University of North Carolina at Chapel Hill, Department of Anthropology. Chapel Hill, NC. \\ 9. University of North Carolina at Chapel Hill, Department of Nutrition. Chapel Hill, NC. \\ 10. Chinese University of Hong Kong. Brain and Mind Institute. Hong Kong. China \\ 11. University of North Carolina Chapel Hill, Department of Psychology and Neuroscience, Chapel \\ Hill, NC. \\ 12. University of North Carolina Chapel Hill, Frank Porter Graham Child Development Institute, \\ Chapel Hill, NC. \\ 13.Michigan State University, Department of Pediatrics and Human Development, East Lansing, \\ MI. \\ 14. Michigan State University, Institute for Quantitative Health Sciences and Engineering, East \\ Lansing, MI.
}

\section{Abstract}

Recently, there has been a surge of interest in the possibility that microbial communities inhabiting the human gut could affect cognitive development and increase risk for mental illness via the "microbiome-gut-brain axis". Infancy likely represents a critical period for the establishment of these relationships, as it is the most dynamic stage of postnatal brain development and a key

Corresponding Authors: Wei Gao, Associate Professor, Biomedical Imaging Research Institute (BIRI), Department of Biomedical Sciences and imaging, Cedars-Sinai Medical Center, PACT Room 400.7S, 116 N Robertson Blvd, Los Angeles, CA 90048, wei.gao@cshs.org, Rebecca Knickmeyer, Associate Professor, Department of Pediatrics and Human Development, Michigan State University, Bioengineering Building, Room 2114, 775 Woodlot Dr., East Lansing, MI 48824, knickmey@ msu.edu. 
period in the maturation of the microbiome. Indeed, recent reports indicate that characteristics of the infant gut microbiome are associated with both temperament and cognitive performance. The neural circuits underlying these relationships have not yet been delineated. To address this gap, resting-state fMRI scans were acquired from 39 one-year-old human infants who had provided fecal samples for identification and relative quantification of bacterial taxa. Measures of alpha diversity were generated and tested for associations with measures of functional connectivity. Primary analyses focused on the amygdala as manipulation of the gut microbiota in animal models alters the structure and neurochemistry of this brain region. Secondary analyses explored functional connectivity of nine canonical resting state functional networks. Alpha diversity was significantly associated with functional connectivity between the amygdala and thalamus and between the anterior cingulate cortex and anterior insula. These regions play an important role in processing/responding to threat. Alpha diversity was also associated with functional connectivity between the supplementary motor area (SMA, representing the sensorimotor network) and the inferior parietal lobule (IPL). Importantly, SMA-IPL connectivity also related to cognitive outcomes at 2 years of age, suggesting a potential pathway linking gut microbiome diversity and cognitive outcomes during infancy. These results provide exciting new insights into the gut-brain axis during early human development and should stimulate further studies into whether microbiome-associated changes in brain circuitry influence later risk for psychopathology.

\section{Introduction}

There has been a recent surge of interest in the possibility that the trillions of microbes inhabiting the human gut (the gut microbiome) influence brain development and risk for mental disorders (Rogers et al., 2016). Studies in rodents have established that manipulations of the gut microbiome alter stress-reactivity, anxiety and depression-like behaviors, as well as social interaction (Carabotti et al., 2015; Rogers et al., 2016). In considering what neural circuits might mediate associations between the gut microbiota and these outcomes, the amygdala has attracted considerable research attention due to its central role in fear (Amaral, 2003; Canteras et al., 2010), stress (Ressler, 2010), emotional regulation (Banks et al., 2007), and social cognition (Adolphs, 2010). Indeed, rodent studies have shown gut microbiota-related alterations in volume (Luczynski et al., 2016), dendritic morphology (Luczynski et al., 2016), monoamine levels (Desbonnet et al., 2010), and gene expression (Bercik et al., 2011; Diaz Heijtz et al., 2011; Neufeld et al., 2011b; Stilling et al., 2015) of the amygdala. Importantly, one of our recent studies (Salzwedel et al., 2018) on human amygdala development during infancy has shown significant associations between one- and two-year amygdala functional connectivity growth and 4 year emotional behavior outcomes, supporting the hypothesis that the amygdala acts as a critical link between the gut and emotional/social behaviors, which are disrupted in a wide range of psychiatric disorders.

In humans, the initial period of postnatal development is critical for microbial colonization of the intestine, which is relatively sterile at birth and rapidly progresses to an adult-like profile by the end of the first year (Backhed et al., 2015) (Koenig et al., 2011). This period is also characterized by rapid structural brain growth (Knickmeyer et al., 2008) and immense functional reconfigurations reflected by the assembly of a variety of functional brain networks(Gao et al., 2009; Smyser et al., 2010; Fransson et al., 2011; Gao et al., 2014 Gao et 
al., 2016; Gilmore et al., 2018). Proper cross-talk between the gut microbiota and the brain during this period may be critical for long-term developmental outcomes as subtle perturbations in either domain could have cascading effects on the other, which are increasingly difficult to reverse with increasing age. Indeed, animal models have shown that recolonization of germ-free mice normalizes anxiety phenotypes at 3 weeks of postnatal age (Clarke et al., 2013) but not at 10 weeks of age (Neufeld et al., 2011a), suggesting a windowing effect. Therefore, a better understanding of the gut-brain axis during early development represents one of the priorities in this line of research.

Previous research in human toddlers suggests that differences in gut microbiome composition, including alpha diversity, beta diversity, and abundances of specific bacterial species, are related to aspects of infant temperament including surgency/extraversion, effortful control, and fear (Christian et al., 2015). This is in line with animal studies and again indicates the critical involvement of amygdala-based emotional/social circuits in gutbrain interactions. In addition to the links between gut microbiome and emotional/social behaviors, prior research by our group (Carlson et al., 2018) has shown that microbial diversity at one year of age is also associated with cognitive performance at two years of age measured using the Mullen Scales of Early learning. Specifically, greater alpha diversity was associated with poorer cognitive performance, an effect that was particularly evident when measuring receptive and expressive language. This suggests that the gut microbiome may also shape cortical circuits associated with cognitive development, however the particular networks involved in this relationship have not yet been identified. In our previous studies (Alcauter et al., 2014a; Alcauter et al., 2014b; Gao et al., 2016) (Salzwedel et al., 2018), functional connectivity properties of the brain's various resting-state functional networks were shown to be correlated with cognitive development scores. Therefore, the potential of microbiome influencing cognitive outcomes through different resting-state functional networks deserves further exploration.

To address these gaps, we performed resting-state functional magnetic resonance imaging (rsfMRI) (Biswal et al., 1995; Gao et al., 2016; Gilmore et al., 2018) on 39 1-year-old infants who had donated fecal samples for the identification and quantification of bacterial taxa. Given previous findings on the involvement of the amygdala in gut-brain interactions in both animal (Diaz Heijtz et al., 2011; Luczynski et al., 2016) and human studies (Christian et al., 2015), our primary analyses focused on functional connectivity of the amygdala. We also carried out a series of exploratory analyses using nine cortical seeds, each representing a canonical resting state network (Smith et al., 2009) in order to identify potential neural circuits underlying previously observed associations between microbial diversity and cognitive outcomes. We hypothesized that there would be significant correlations between gut microbiome diversity and amygdala functional connectivity in human infants. Moreover, we also expected to detect significant microbiome-functional connectivity relationships among other cortical functional networks that are essential for cognition(Carlson et al., 2018). 


\subsection{Methods}

\subsection{Subjects.}

Infants were drawn from the UNC Early Brain Development Study, an ongoing prospective longitudinal study of early brain development being carried out at the University of North Carolina at Chapel Hill (Gilmore et al., 2007; Knickmeyer et al., 2008; Gilmore et al., 2010; Gao et al., 2014; Gao et al., 2016; Knickmeyer et al., 2017; Carlson et al., 2018; Gao et al., 2018; Gilmore et al., 2018) To be included in the present study infants were required to have fecal samples as well as usable resting state functional and structural magnetic resonance imaging data. Initial microbiome characterization was conducted using a larger crosssectional sample of one-year old infants $(n=89)$ with fecal samples. 39 of these infants also completed structural MRI and resting-state fMRI scans (success rate of $44 \%$ ). The other 50 children either did not go to sleep or woke up before scanning was complete. The final microbiome-functional connectivity analyses were conducted using the 39 infants with fecal samples and usable structural / functional imaging data. Informed written consent was obtained from a parent or legal guardian. This study was approved by the Institutional Review Board of the University of North Carolina at Chapel Hill.

2.1.1 Infant microbiome analysis.-Participating families were provided with a sample collection kit at the infant's 1-year MRI visit. Parents were instructed to collect approximately $200 \mathrm{mg}$ of feces from a single diaper, immediately submerge in a tube containing Allprotect reagent (Qiagen cat \# 76405), and mail it back through overnight mail (samples can be stored up to 7 days at $15-25^{\circ} \mathrm{C}$ ). Once received, the tubes were stored at $-80^{\circ} \mathrm{C}$ until analysis. The MiSeq platform (Illumina, San Diego, CA) was used for $16 \mathrm{~S}$ rRNA amplicon sequencing of the $\mathrm{V} 1-\mathrm{V} 2$ gene region and subsequent identification and relative quantification of bacterial taxa. Quantitative Insights Into Microbial Ecology (QIIME) (Lozupone and Knight, 2005; Lozupone et al., 2006) software was used for operational taxonomic unit picking via the de novo algorithm and chimeric sequences were removed with ChimeraSlayer. For the current study, QIIME was used to produce four measures of alpha diversity (Shannon Index (SHAN), observed species (OS), Faith's Phylogenetic Diversity (PD), and Chao1), which were used as the primary predictors of interest. All of these are measures of within individual diversity. Observed species is simply the observed number of OTUs per sample. Chao 1 is an estimate of the total number of OTUs that would be observed with infinite sampling. Shannon Index is a measure of richness and evenness. Finally, Faith's Phylogenetic Diversity is a phylogenetic measure of taxon richness and is expressed as number of tree units observed in the sample. (Navas-Molina 2013). (Additional Details in Supplemental Methods and Materials.)

2.1.2 Cognitive Testing.-The Mullen Scales of Early Learning (Mullen, 1995) were used to measure cognitive development status at 2 years of age. The Mullen includes five separate scales including gross motor, fine motor, visual reception, expressive language, and receptive language, each with their own age-group standardized normative $\mathrm{T}$ scores and percentiles. An Early Learning (Cognitive) Composite (ELC) similar to an IQ score or the Bayley Mental Development Index can also be derived. The standardized T scores of four scales (gross motor not included) are combined to create the ELC. The Mullen has good 
standardization and reliability data, with median internal consistency scores ranging between 0.75 and 0.91 , and test-retest correlations over 0.82 for 1 to 25 months. Mullen data were collected over a period of 3 years by several experienced examiners who were blind to microbiome characteristics. All cognitive data were screened for quality control and no data warranted exclusion from this study (see Supporting Materials-Quality Control for Cognitive Testing). This provided cognitive data for 30 subjects at 2 years of age.

2.2.3 Participant Characteristics and Covariate Identification.-In order to detect associations between gut microbiome properties, functional connectivity and behavior, proper control of covariates is essential. In this study, the pool of potential covariates included different participant characteristics (Table 1) extracted from either parental report (PR) or medical record review (MRR): income (PR; rank based on poverty level for the household size at the year of visit; low $<200 \%$, middle $200 \%-400 \%$, and high $>400 \%$ federal poverty level), maternal/paternal psychosis [PR or MRR positive for psychiatric history of schizophrenia spectrum, bipolar, depressive, anxiety, attention-deficit/ hyperactivity, and/or autism spectrum disorders as well as Tourette's syndrome), gestational age at birth (MRR; days), postnatal age at scan ((MRR, days), birth weight (MRR; grams), $>24 \mathrm{hr}$ stay in neonatal intensive care (MRR), APGAR scores at 5 minutes post-birth (MRR), maternal/paternal age (PR), maternal/paternal education (PR; years), surgical anesthesia (MRR), older siblings (PR \& MRR), currently breast feeding (PR), currently on formula (PR), ever on formula (PR), sex (MRR), delivery method (vaginal or Caesarian; MRR), receipt of milk other than breast milk or formula (cow or goat; PR), kind of milk received other than breast milk or formula (none, cow, or goat; PR), illness in the week preceding MRI (PR), gastrointestinal symptoms in the week preceding MRI (PR), antibiotics during the first year of postnatal life (PR or MRR; oral antibiotics count as positive finding), and maternal use of antibiotics during pregnancy (MRR).

We sought to minimize the possibility that any observed associations were secondary to known confounding factors. In our previous study, Fisher Exact Test (categorical variables) and linear mixed effect modeling (continuous variables) were used to identify the following covariates related to microbiome alpha diversity measurements (Carlson et al., 2018): paternal ethnicity and older siblings. Covariates related to 2-year cognitive outcome variables were also identified in a larger sample via adaptive lasso; these included sex, maternal education, paternal age, paternal ethnicity, twin status, and income. Finally, several covariates were shown to be related to 1-year global brain volumes via adaptive lasso (Knickmeyer et al., 2017; Carlson et al., 2018): birth weight, sex, postnatal age at scan, twin status, maternal education, and paternal education. Given that no previous studies have systematically examined covariates for the brain's functional connectivity at 1 year of age, we decided to use this set of covariates related to the brain volume as surrogate covariates for our functional connectivity related analyses. Therefore, when testing FC $\sim$ gut microbiome associations, covariates for both alpha diversity and FC were included, whereas testing for $\mathrm{FC} \sim$ cognition relationships, any remaining unique covariates were also included (i.e. income and paternal age). Additionally, residual frame-wise displacement after motion scrubbing was included as a covariate in all models. 


\subsection{Imaging and Image Preprocessing}

2.2.1 Imaging.-Before imaging, each participant was fed, swaddled, and fitted with ear protection. Subjects slept during image acquisition without sedation. Head position was secured in the scanner using a vacuum-fixation device. Infant images were acquired using a 3T Siemens Tim Trio with 32-channel head coil. Functional images were acquired using a $T_{2}{ }^{*}$-weighted EPI sequence: time repetition $(\mathrm{TR})=2 \mathrm{~s}$, time echo $(\mathrm{TE})=32 \mathrm{~ms}, 33$ slices, voxel size of $4 \mathrm{~mm}^{3}, 150$ volumes. Structural images were acquired using a 3D Magnetization Prepared Rapid Acquisition Gradient-Recalled Echo sequence (MPRAGE: $\mathrm{TR}=1900 \mathrm{~ms}, \mathrm{TE}=3.74 \mathrm{~ms}$, flip angle $=7^{\circ}$,), with a voxel size of $1 \mathrm{~mm}^{3}$.

2.2.2 Image preprocessing.-Functional data were preprocessed using two software packages: FMRIB Software Library (FSL v4.1.9) and the Analysis of Functional NeuroImages suite (AFNI v2011-12-21-1014). Steps included discarding the first 10 volumes, slice-timing correction, rigid-body motion correction, bandpass filtering (0.01$0.08 \mathrm{~Hz}$ ), and regression of whole brain [global signal regression (GSR)], white matter, CSF, and the six motion parameters. Data scrubbing was also implemented; scrubbing criteria, $0.5 \%$ signal change and $0.5 \mathrm{~mm}$ frame-wise displacement (FD). A threshold of 90 volumes remaining was used as the criterion for subject exclusion due to motion artifacts but none of the 39 subjects was excluded. Alignment of functional data into a common space involved two steps: (1) within-subject rigid alignment [FSL FLIRT (for FMRIB Linear Image Restoration Tool)] between functional and T1-weighted images; and (2) nonlinear [FSL FNIRT (for FMRIB Nonlinear Image Registration Tool)] registration of the T1-weighted images to a 1-year template (Shi et al,. 2011). The combined transformation field (linear plus nonlinear) was used to warp the preprocessed rsfMRI data to the template space.

\subsection{Data Analyses and Statistics}

\subsubsection{Association between microbiome and amygdala functional}

connectivity.-In this study we were primarily interested in the relationship between alpha diversity (PD, SHAN, OS, and Chao1) and functional connectivity associated with the amygdala. First, we used the seed-based approach to generate voxel-wise temporal correlation measures (i.e., functional connectivity) (Biswal et al., 1995) for the left and right amygdala using anatomically defined ROIs defined in the 1-year infant-specific brain atlas (Shi et al., 2011). Then, for each voxel we used analysis of covariance (ANCOVA) to test for microbiome related effects. In the ANCOVA we modeled the Fisher's Z-transformed correlation measure as the response variable and one of four microbiome alpha diversity as the main-effect. Covariates in the alpha diversity related computations included older sibling, paternal ethnicity, birth weight, post-natal age at scan, sex, twin status, maternal/ paternal education, and residual FD. Based on recent guidelines for multiple comparison correction at the whole brain level (Eklund et al., 2016), significant effects were defined using a voxel-wise $\mathrm{P}<0.001$ and a cluster size threshold of 7 face-connected voxels (AFNI; $3 \mathrm{dClustSim}, a<0.05$ ). Note these settings were computed using a non-gaussian or mixed model (gaussian plus mono-exponential) auto-correlation function and smoothness estimates from the data (AFNI; 3dFWHMx). Post-hoc cluster level responses were generated using the average FC per subject for each cluster detected. 
2.3.2 Association between functional connectivity and behavior.-Next, for those significant clusters $(\alpha<0.05)$ demonstrating functional connectivity $\sim$ alpha diversity relationships we further explored the association between FC and MSEL using post-hoc correlation analyses. Briefly, the average FC for each cluster/subject at 1-year served as the predictor variable with Mullen ELC at 2-years as the outcome measure. Here we further included paternal age and income as additional covariates given their association with MSEL at this time point (Carlson et al., 2018).

\subsubsection{Association between microbiome and functional connectivity for other} brain regions.-To further explore microbiome-functional connectivity-behavior relationships in other brain regions, nine seeds were generated based on adult resting-state networks (Smith et al., 2009) warped into the 1-year old template space (Shi et al., 2011). These seed-regions, defined as 6-mm spheres at network maxima (see Tbl. S1 for coordinates), were used to conduct similar microbiome $\sim$ FC and FC-behavior analyses - see sections 2.3.1 \& 2.3.2. Identical statistical thresholding procedures as those in amygdalabased analyses were used for this set of seeds.

\subsection{Results}

\subsection{Amygdala-mid/forebrain functional connectivity correlates with alpha diversity in one- year olds.}

Our initial analysis focused on detecting microbiome-amygdala functional connectivity (FC) relationships at 1-year of age. We identified one significant cluster within the thalamic regions (Fig. 1a; cluster size $=18$ voxels and center-of-mass: $x=-2.3, y=12.8, z=5$ ) that had a significant main-effect of microbiome alpha diversity (Faith's Phylogenetic Diversity (PD)) on the left amygdala functional connectivity (Shi et al., 2011). Post-hoc cluster-level analysis revealed a strong linear relationship between PD and left amygdala FC in this region (Fig. 1b; $r=-0.55, P<0.001$ ). Similar relationships were observed for the other alpha diversity measures within this cluster; SHAN: $\mathrm{r}=-0.36, \mathrm{P}=0.023$; OS $\mathrm{r}=-0.40, \mathrm{P}=$ 0.010 ; and Chao1 $\mathrm{r}=-0.33, \mathrm{P}=0.040$. However, $\mathrm{FC}$ associated with this cluster was not significantly related to ELC at 2-years.

\subsection{Anterior cingulate-insular and sensorimotor-parietal functional connectivity (FC) correlates with alpha diversity in one-year olds and sensorimotor-parietal FC is associated with 2-year cognitive outcomes.}

Expanding the analyses to include other brain regions [i.e. using seeds regions based on resting-state networks (Smith et al., 2009) warped into 1-year template space (Shi et al., 2011) - see Table S1] we detected two additional clusters showing significant $(a=0.05)$ FC $\sim$ alpha diversity relationships. First, connectivity between the anterior cingulate cortex (ACC) and right anterior insula [Fig. $2 \mathrm{a}$; cluster size $=14$ voxels and center-of-mass: $\mathrm{x}=$ $-33.3, y=-20.1, z=2.4)$ demonstrated a main-effect for Chao1. Here correlation analyses revealed a negative relationship between FC and alpha diversity (Fig. 2b; r $=-0.54$ and $\mathrm{P}<$ 0.001). Similar relationships were observed for the other alpha diversity measures within this cluster; PD: $r=-0.31, \mathrm{P}=0.060$; SHAN: $\mathrm{r}=-0.42, \mathrm{P}=0.009$; OS $\mathrm{r}=-0.40, \mathrm{P}=0.020$. Moreover, FC between supplemental motor area (SMA) and the left parietal cortex [inferior 
parietal lobule (IPL), Fig. 3a; cluster size $=8$ voxels and center-of-mass: $x=40.5, y=41$, $\mathrm{z}=44.5$ ] exhibited a main-effect of alpha diversity for OS, with subsequent correlation analyses revealing a positive relationship (Fig $3 b$; OS: $r=0.41, P=0.009$ ). Correlations with other diversity measures were again similar: PD: $r=0.35, P=0.031$; SHAN: $r=0.29, P=$ 0.070; and Chao1: $r=0.38, P=0.020$. Finally, $F C$ associated with this cluster was associated with Mullen ELC at 2-years (Fig. 3c; $r=-0.42, \mathrm{P}=0.049$ ).

\section{Discussion}

The amygdala is a key node in the neural circuit that allows humans and animals to learn, detect, and respond to threats (Canteras et al., 2010). Germ-free mice, which are microbiota deficient from birth, exhibit increased amygdala volume (Luczynski et al., 2016), dendritic hypertrophy in the basolateral amygdala (Luczynski et al., 2016), and changes in gene expression that suggest increased neural activity of the amygdala (Stilling et al., 2015). These changes are accompanied by altered stress hormone signaling and anxiety-like behaviors, as well as deficits in social cognition (Carabotti et al., 2015; Rogers et al., 2016). In this preliminary study of 39 one-year-old human infants, we found evidence of associations between the gut microbiome and functional connectivity of the amygdala, suggesting similar mechanisms are at play during human brain development. Specifically, we observed significant negative associations between 1-year microbiome diversity measures and connectivity between the left amygdala and mid/forebrain regions that overlapped with the thalamus. Thalamic connections with the amygdala likely relay critical sensory information to the amygdala for initial emotional appraisal (Jones, 2000). Given well-documented effects of the gut microbiome on anxiety-related behaviors in rodents, it is particularly interesting to note that the paraventricular nucleus of the thalamus (PVT) is strongly connected to the lateral division of the central amygdala, and this circuit plays a key role in fear processing (Penzo et al., 2015) and negative emotionality (Li et al., 2010). Therefore, the negative correlations observed here (i.e., higher levels of microbiome diversity is related to weaker thalamusamygdala connectivity) suggest that increased levels of gut microbiome diversity in 1-year olds infants may be associated with less efficient fear/ emotion processing. In this study, the lateralized findings centered on the left amygdala is intriguing and consistent with our previous reports showing that prenatal drug exposures also affect the left amygdala more than the right (Grewen et al., 2015; Salzwedel et al., 2015). These findings may be related to the functional bias of the left amygdala toward sustained emotional processing (Baas et al., 2004; Wagner et al., 2006) but future studies are needed to validate and more specifically address this phenomenon.

Beyond the amygdala, results from our exploratory analysis of canonical brain networks also implicated effects of the microbiome in circuits relevant to fear and anxiety. Specifically, we observed significant negative associations between one-year microbiome diversity measures and connectivity between anterior cingulate cortex and the right anterior insula. The anterior insula acts a salience control center that weighs the importance of both internal and external signals, including those related to threat, for proper attention allocation and behavioral switching (Menon and Uddin, 2010). The cingulate cortex has long been thought to play a central role in emotional processing (Papez, 1995), with the dorsal anterior cingulate (dACC) and adjacent dorsomedial prefrontal cortex (dmPFC) playing a specific role in the 
appraisal and expression of fear (Kalisch and Gerlicher, 2014). The emergence of fearrelated processing in infancy is normal and critical for survival and well-being (Jessen and Grossmann, 2016). However, high levels of fear-reactivity and heightened attention to fearrelevant stimuli are associated with increased vulnerability to later anxiety disorders (Shin and Liberzon, 2010). Notably, insula hyper-reactivity to threat has been proposed to play a key role in social anxiety disorder(Duval et al., 2015), and this hyper-reactivity may be related to the altered connectivity between the anterior insula and anterior cingulate observed in this study. Indeed, Klumpp et al (Klumpp et al., 2012) have reported that greater insula reactivity during a fear processing task is related to reduced functional connectivity between the insula and the dorsal anterior cingulate cortex. Although the current study did not include direct measures of fear reactivity and negative emotionality, taken together, the observed significant correlations between gut microbiome diversity and two of the brain's essential emotion regulation circuits (i.e., amygdala-thalamus and insula-ACC) suggest that the microbiome likely influences anxiety-related behaviors during infancy. Future studies with direct emotional/social behavioral measures (e.g., temperament) are needed to explicitly test this hypothesis.

Finally, we also observed positive associations between alpha diversity and functional connectivity between the supplementary motor area (SMA, representing the sensorimotor network) and the inferior parietal lobule (IPL), a multi-modal association area linking auditory, visual, and somatosensory cortices (Catani and ffytche, 2005). This area also has extensive connections to Broca's area and Wernicke's area, classic components in the language-processing network(Catani and ffytche, 2005). The IPL is likely a crucial node in the fronto-parietal system responsible for sustaining attention, detecting salient or novel events, phasic alerting and switching between task-sets (Singh-Curry and Husain, 2009). These foundational abilities are expected to support cognitive development and language acquisition. In keeping with this hypothesis, SMA-IPL connectivity at 1 year of age was related to cognitive outcomes at 2 years of age (Fig. 3C), providing potential pathways linking gut microbiome diversity and cognitive outcomes during infancy (Carlson et al., 2018). This finding suggest that early variation in functional connectivity may induce developmental cascades that ultimately influence later cognitive outcomes. Indeed, the idea that function in one domain or level or system influences another system or level of function over time, resulting in spreading effects, is widely accepted in developmental science (Masten and Cicchetti, 2010). Our own prior work demonstrating that amygdala functional connectivity growth during the second year is associated with 4-year emotional and cognitive developmental outcomes is in keeping with this model (Salzwedel et al. 2018), as is our prior study demonstrating that 1-year-old thalamus-salience network connectivity significantly predicts working memory performance in 2-year-olds (Alcauter et al, 2014b).

Limitations of the current study include the small sample size, the lack of developmental measures directly related to amygdala function (e.g., temperament and social cognition), and the assessment of the microbiota at a single time point. In the future, studies with larger sample sizes are needed to confirm the current findings with potentially more stringent corrections of multiple corrections that take into account not only whole-brain voxel-wise corrections, as conducted in this study, but also the number of diversity measures and functional connectivity metrics evaluated. Moreover, additional studies with dense sampling 
of microbiome, brain, and behavior are needed to elucidate the potentially intricate relationships between these different domains across development. Such studies could also incorporate additional potential confounding variables which were not available for the current including direct measured of maternal stress and the type and diversity of solid foods. Further, microbiome composition may be important in addition to diversity. Our previous work indicates that one-year-old infants can be clustered into three enterotypes based on the relative abundance of different bacterial genera. One cluster is characterized by high levels of Faecalibacterium, one by high levels of Bacteroides, and one by high levels of an unclassified genus of Ruminococcaceae. However, the numbers of children in each cluster were not sufficient for us to examine relations with functional connectivity in this study. Nonetheless, the current study provides important, new information about the microbiome-gut-brain axis in human infants.

In conclusion, this study provides initial evidence that the diversity of the gut microbiome is associated with the integrity of functional neural circuits during early human development. The circuits detected are critical for fear-processing and cognitive development, both of which are associated with later vulnerability to psychopathology (Degnan and Fox, 2007; Hirshfeld-Becker et al., 2008; Gartstein et al., 2010). Therefore, these findings may have important implications for improving mental health. Future studies should determine whether microbiome-associated changes in neurocircuitry influence later risk for psychopathology, particularly in the realm of anxiety-related behaviors. It would also be of interest to determine whether differences in functional connectivity are mirrored by differences in anatomical connectivity measured by diffusion tensor imaging, particularly given the reported hypermyelination of prefrontal cortex in germ-free mice (Hoban et al., 2016). Ultimately, modulation of the gut microbiota may be a tractable strategy for preventing or ameliorating disorders characterized by altered processing of threat or delayed cognitive development.

\section{Supplementary Material}

Refer to Web version on PubMed Central for supplementary material.

\section{Acknowledgement:}

RCK is a co-investigator and WG is a consultant on a grant sponsored by Nestle/Wyeth (RDNN201704/4520562240); RCK has also received travel support to present at the 7th Annual Wyeth Nutrition Science Center Global Summit. The other authors declare no competing financial interests. This work was supported by National Institutes of Health (R01DA042988, R01DA043678, R21NS088975, R21DA043171, R03DA036645 to WG; R01MH070890 and R01HD053000 to JHG; R01 MH092335 and R33MH104330 to RKS; T32 NS007432 to ALC), and Cedars-Sinai Precision Medicine Initiative Award and institutional support to WG. Jennifer Prater was the lead study coordinator with assistance from Dianne Evans and Wendy Neuheimer. We are grateful to the research assistants collecting 2-year cognitive data: Margaret Hamilton Fox, Mallory Turner, Margo Williams, Haley Parrish Black, and Emma Brink. Joe Blocher and Rachel Steiner at the Neuro Image Research and Analysis Laboratories provided image processing support.

\section{References:}

Adolphs R (2010) What does the amygdala contribute to social cognition? Annals of the New York Academy of Sciences 1191:42-61. [PubMed: 20392275] 
Alcauter S, Lin W, Smith JK, Goldman BD, Reznick JS, Gilmore JH, Gao W (2014a) Frequency of spontaneous BOLD signal shifts during infancy and correlates with cognitive performance. Dev Cogn Neurosci 12C:40-50.

Alcauter S, Lin W, Smith JK, Short SJ, Goldman BD, Reznick JS, Gilmore JH, Gao W (2014b) Development of Thalamocortical Connectivity during Infancy and Its Cognitive Correlations. J Neurosci 34:9067-9075. [PubMed: 24990927]

Amaral DG (2003) The amygdala, social behavior, and danger detection. Ann N Y Acad Sci 1000:337-347. [PubMed: 14766647]

Baas D, Aleman A, Kahn RS (2004) Lateralization of amygdala activation: a systematic review of functional neuroimaging studies. Brain Res Brain Res Rev 45:96-103. [PubMed: 15145620]

Backhed F et al. (2015) Dynamics and Stabilization of the Human Gut Microbiome during the First Year of Life. Cell Host Microbe 17:852. [PubMed: 26308884]

Banks SJ, Eddy KT, Angstadt M, Nathan PJ, Phan KL (2007) Amygdala-frontal connectivity during emotion regulation. Soc Cogn Affect Neurosci 2:303-312. [PubMed: 18985136]

Bercik P, Denou E, Collins J, Jackson W, Lu J, Jury J, Deng Y, Blennerhassett P, Macri J, McCoy KD, Verdu EF, Collins SM (2011) The intestinal microbiota affect central levels of brain-derived neurotropic factor and behavior in mice. Gastroenterology 141:599-609, 609 e591-593. [PubMed: 21683077]

Biswal B, Yetkin FZ, Haughton VM, Hyde JS (1995) Functional connectivity in the motor cortex of resting human brain using echo-planar MRI. Magn Reson Med 34:537-541. [PubMed: 8524021]

Canteras NS, Resstel LB, Bertoglio LJ, Carobrez Ade P, Guimaraes FS (2010) Neuroanatomy of anxiety. Curr Top Behav Neurosci 2:77-96. [PubMed: 21309107]

Carabotti M, Scirocco A, Maselli MA, Severi C (2015) The gut-brain axis: interactions between enteric microbiota, central and enteric nervous systems. Ann Gastroenterol 28:203-209. [PubMed: 25830558]

Carlson AL, Xia K, Azcarate-Peril MA, Goldman BD, Ahn M, Styner MA, Thompson AL, Geng X, Gilmore JH, Knickmeyer RC (2018) Infant Gut Microbiome Associated With Cognitive Development. Biol Psychiatry 83:148-159. [PubMed: 28793975]

Catani M, ffytche DH (2005) The rises and falls of disconnection syndromes. Brain 128:2224-2239. [PubMed: 16141282]

Christian LM, Galley JD, Hade EM, Schoppe-Sullivan S, Kamp Dush C, Bailey MT (2015) Gut microbiome composition is associated with temperament during early childhood. Brain Behav Immun 45:118-127. [PubMed: 25449582]

Clarke G, Grenham S, Scully P, Fitzgerald P, Moloney RD, Shanahan F, Dinan TG, Cryan JF (2013) The microbiome-gut-brain axis during early life regulates the hippocampal serotonergic system in a sex-dependent manner. Molecular psychiatry 18:666-673. [PubMed: 22688187]

Degnan KA, Fox NA (2007) Behavioral inhibition and anxiety disorders: multiple levels of a resilience process. Dev Psychopathol 19:729-746. [PubMed: 17705900]

Desbonnet L, Garrett L, Clarke G, Kiely B, Cryan JF, Dinan TG (2010) Effects of the probiotic Bifidobacterium infantis in the maternal separation model of depression. Neuroscience 170:11791188. [PubMed: 20696216]

Diaz Heijtz R, Wang S, Anuar F, Qian Y, Bjorkholm B, Samuelsson A, Hibberd ML, Forssberg H, Pettersson S (2011) Normal gut microbiota modulates brain development and behavior. Proc Natl Acad Sci U S A 108:3047-3052. [PubMed: 21282636]

Duval ER, Javanbakht A, Liberzon I (2015) Neural circuits in anxiety and stress disorders: a focused review. Ther Clin Risk Manag 11:115-126. [PubMed: 25670901]

Eklund A, Nichols TE, Knutsson H (2016) Cluster failure: Why fMRI inferences for spatial extent have inflated false-positive rates. Proc Natl Acad Sci U S A 113:7900-7905. [PubMed: 27357684]

Fransson P, Aden U, Blennow M, Lagercrantz H (2011) The functional architecture of the infant brain as revealed by resting-state fMRI. Cereb Cortex 21:145-154. [PubMed: 20421249]

Gao W, Lin W, Grewen K, Gilmore JH (2016) Functional Connectivity of the Infant Human Brain: Plastic and Modifiable. Neuroscientist.

Gao W, Alcauter S, Smith J, Gilmore J, W L (2014 ) Development of Human Brain Cortical Network Architecture during Infancy. Brain Structure and Function Doi: 10.1007/s00429-014-0710-3. 
Gao W, Zhu H, Giovanello KS, Smith JK, Shen D, Gilmore JH, Lin W (2009) Evidence on the emergence of the brain's default network from 2-week-old to 2-year-old healthy pediatric subjects. Proc Natl Acad Sci U S A 106:6790-6795. [PubMed: 19351894]

Gao W, Alcauter S, Elton A, Hernandez-Castillo CR, Smith JK, Ramirez J, Lin W (2014) Functional Network Development During the First Year: Relative Sequence and Socioeconomic Correlations. Cereb Cortex.

Gao W, Grewen K, Knickmeyer RC, Qiu A, Salzwedel A, Lin W, Gilmore JH (2018) A review on neuroimaging studies of genetic and environmental influences on early brain development. Neuroimage.

Gartstein MA, Bridgett DJ, Rothbart MK, Robertson C, Iddins E, Ramsay K, Schlect S (2010) A latent growth examination of fear development in infancy: contributions of maternal depression and the risk for toddler anxiety. Dev Psychol 46:651-668. [PubMed: 20438177]

Gilmore JH, Knickmeyer RC, Gao W (2018) Imaging structural and functional brain development in early childhood. Nat Rev Neurosci 19:123-137. [PubMed: 29449712]

Gilmore JH, Schmitt JE, Knickmeyer RC, Smith JK, Lin W, Styner M, Gerig G, Neale MC (2010) Genetic and environmental contributions to neonatal brain structure: A twin study. Hum Brain Mapp 31:1174-1182. [PubMed: 20063301]

Gilmore JH, Lin W, Prastawa MW, Looney CB, Vetsa YS, Knickmeyer RC, Evans DD, Smith JK, Hamer RM, Lieberman JA, Gerig G (2007) Regional gray matter growth, sexual dimorphism, and cerebral asymmetry in the neonatal brain. J Neurosci 27:1255-1260. [PubMed: 17287499]

Grewen K, Salzwedel AP, Gao W (2015) Functional Connectivity Disruption in Neonates with Prenatal Marijuana Exposure. Front Hum Neurosci 9:601. [PubMed: 26582983]

Hirshfeld-Becker DR, Micco J, Henin A, Bloomfield A, Biederman J, Rosenbaum J (2008) Behavioral inhibition. Depression and Anxiety 25:357-367. [PubMed: 18412062]

Hoban AE, Stilling RM, Ryan FJ, Shanahan F, Dinan TG, Claesson MJ, Clarke G, Cryan JF (2016) Regulation of prefrontal cortex myelination by the microbiota. Transl Psychiatry 6:e774. [PubMed: 27045844]

Jessen S, Grossmann T (2016) The developmental emergence of unconscious fear processing from eyes during infancy. J Exp Child Psychol 142:334-343. [PubMed: 26493612]

Jones E (2000) The thalamus. Cambridge: Cambridge University.

Kalisch R, Gerlicher AM (2014) Making a mountain out of a molehill: on the role of the rostral dorsal anterior cingulate and dorsomedial prefrontal cortex in conscious threat appraisal, catastrophizing, and worrying. Neurosci Biobehav Rev 42:1-8. [PubMed: 24525267]

Klumpp H, Angstadt M, Phan KL (2012) Insula reactivity and connectivity to anterior cingulate cortex when processing threat in generalized social anxiety disorder. Biol Psychol 89:273-276. [PubMed: 22027088]

Knickmeyer RC, Xia K, Lu Z, Ahn M, Jha SC, Zou F, Zhu H, Styner M, Gilmore JH (2017) Impact of Demographic and Obstetric Factors on Infant Brain Volumes: A Population Neuroscience Study. Cereb Cortex 27:5616-5625. [PubMed: 27797836]

Knickmeyer RC, Gouttard S, Kang C, Evans D, Wilber K, Smith JK, Hamer RM, Lin W, Gerig G, Gilmore JH (2008) A structural MRI study of human brain development from birth to 2 years. J Neurosci 28:12176-12182. [PubMed: 19020011]

Koenig JE, Spor A, Scalfone N, Fricker AD, Stombaugh J, Knight R, Angenent LT, Ley RE (2011) Succession of microbial consortia in the developing infant gut microbiome. Proc Natl Acad Sci U S A 108 Suppl 1:4578-4585. [PubMed: 20668239]

Li Y, Li S, Wei C, Wang H, Sui N, Kirouac GJ (2010) Changes in emotional behavior produced by orexin microinjections in the paraventricular nucleus of the thalamus. Pharmacol Biochem Behav 95:121-128. [PubMed: 20045021]

Lozupone C, Knight R (2005) UniFrac: a new phylogenetic method for comparing microbial communities. Appl Environ Microbiol 71:8228-8235. [PubMed: 16332807]

Lozupone C, Hamady M, Knight R (2006) UniFrac--an online tool for comparing microbial community diversity in a phylogenetic context. BMC Bioinformatics 7:371. [PubMed: 16893466] 
Luczynski P, Whelan SO, O’Sullivan C, Clarke G, Shanahan F, Dinan TG, Cryan JF (2016) Adult microbiota-deficient mice have distinct dendritic morphological changes: differential effects in the amygdala and hippocampus. Eur J Neurosci.

Masten AS, Cicchetti D (2010) Developmental cascades. Dev Psychopathol 22:491-495. [PubMed: 20576173]

Mullen EM (1995) Mullen Scales of Early Learning. Circle Pines, MN: American Guidance Service, Inc.

Neufeld KA, Kang N, Bienenstock J, Foster JA (2011a) Effects of intestinal microbiota on anxiety-like behavior. Commun Integr Biol 4:492-494. [PubMed: 21966581]

Neufeld KM, Kang N, Bienenstock J, Foster JA (2011b) Reduced anxiety-like behavior and central neurochemical change in germ-free mice. Neurogastroenterol Motil 23:255-264, e119. [PubMed: 21054680]

Papez JW (1995) A proposed mechanism of emotion. 1937. J Neuropsychiatry Clin Neurosci 7:103112. [PubMed: 7711480]

Penzo MA, Robert V, Tucciarone J, De Bundel D, Wang M, Van Aelst L, Darvas M, Parada LF, Palmiter RD, He M, Huang ZJ, Li B (2015) The paraventricular thalamus controls a central amygdala fear circuit. Nature 519:455-459. [PubMed: 25600269]

Ressler KJ (2010) Amygdala activity, fear, and anxiety: modulation by stress. Biol Psychiatry 67:1117-1119. [PubMed: 20525501]

Rogers GB, Keating DJ, Young RL, Wong ML, Licinio J, Wesselingh S (2016) From gut dysbiosis to altered brain function and mental illness: mechanisms and pathways. Molecular psychiatry 21:738-748. [PubMed: 27090305]

Salzwedel AP, Grewen KM, Vachet C, Gerig G, Lin W, Gao W (2015) Prenatal drug exposure affects neonatal brain functional connectivity. J Neurosci 35:5860-5869. [PubMed: 25855194]

Salzwedel AP, Stephens RL, Goldman BD, Lin W, Gilmore JH, Gao W (2018) Development of Amygdala Functional Connectivity During Infancy and Its Relationship With 4-Year Behavioral Outcomes. Biol Psychiatry Cogn Neurosci Neuroimaging.

Shi F, Yap PT, Wu G, Jia H, Gilmore JH, Lin W, Shen D (2011) Infant brain atlases from neonates to 1- and 2-year-olds. PLoS One 6:e18746. [PubMed: 21533194]

Shin LM, Liberzon I (2010) The neurocircuitry of fear, stress, and anxiety disorders. Neuropsychopharmacology 35:169-191. [PubMed: 19625997]

Singh-Curry V, Husain M (2009) The functional role of the inferior parietal lobe in the dorsal and ventral stream dichotomy. Neuropsychologia 47:1434-1448. [PubMed: 19138694]

Smith SM, Fox PT, Miller KL, Glahn DC, Fox PM, Mackay CE, Filippini N, Watkins KE, Toro R, Laird AR, Beckmann CF (2009) Correspondence of the brain's functional architecture during activation and rest. Proc Natl Acad Sci U S A 106:13040-13045. [PubMed: 19620724]

Smyser CD, Inder TE, Shimony JS, Hill JE, Degnan AJ, Snyder AZ, Neil JJ (2010) Longitudinal analysis of neural network development in preterm infants. Cereb Cortex 20:2852-2862. [PubMed: 20237243]

Stilling RM, Ryan FJ, Hoban AE, Shanahan F, Clarke G, Claesson MJ, Dinan TG, Cryan JF (2015) Microbes \& neurodevelopment--Absence of microbiota during early life increases activity-related transcriptional pathways in the amygdala. Brain, behavior, and immunity 50:209-220.

Wagner G, Koch K, Reichenbach JR, Sauer H, Schlosser RG (2006) The special involvement of the rostrolateral prefrontal cortex in planning abilities: an event-related fMRI study with the Tower of London paradigm. Neuropsychologia 44:2337-2347. [PubMed: 16806311] 
a

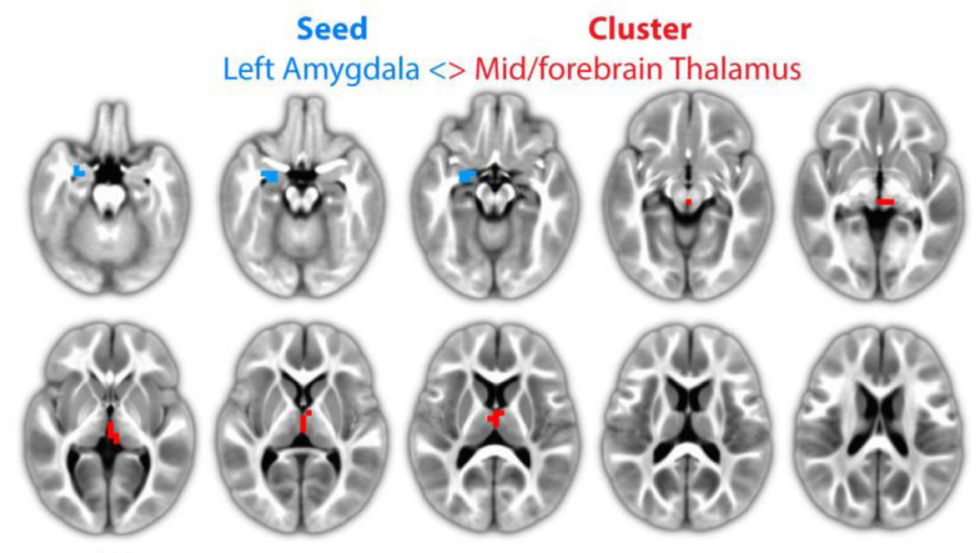

b

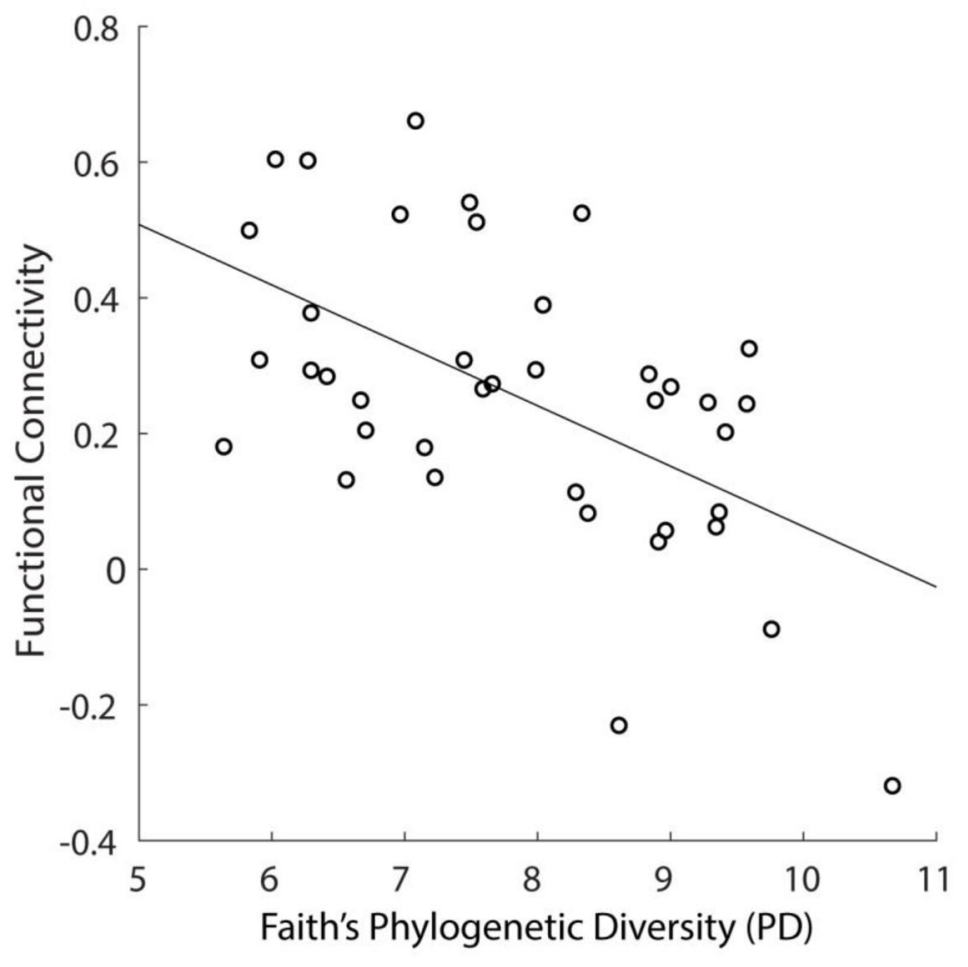

Figure 1:

Functional connectivity between the left amygdala (seed-region, blue color) and mid/ forebrain (cluster localization) correlates with alpha diversity in one-year olds. (a) Visualization of significant cluster $(a<0.05$; voxel-wise $\mathrm{P}<0.001$ and $7+$ face-connected voxels) located primarily within the thalamic region. (b) Corresponding cluster-level plot of average functional connectivity versus microbiome alpha diversity (Faiths Phylogenetic Diversity). Data-points correspond to individual subjects $(\mathrm{N}=39)$. Line shows best linear fit; $\mathrm{r}=-0.55, \mathrm{P}<0.001)$. 
a

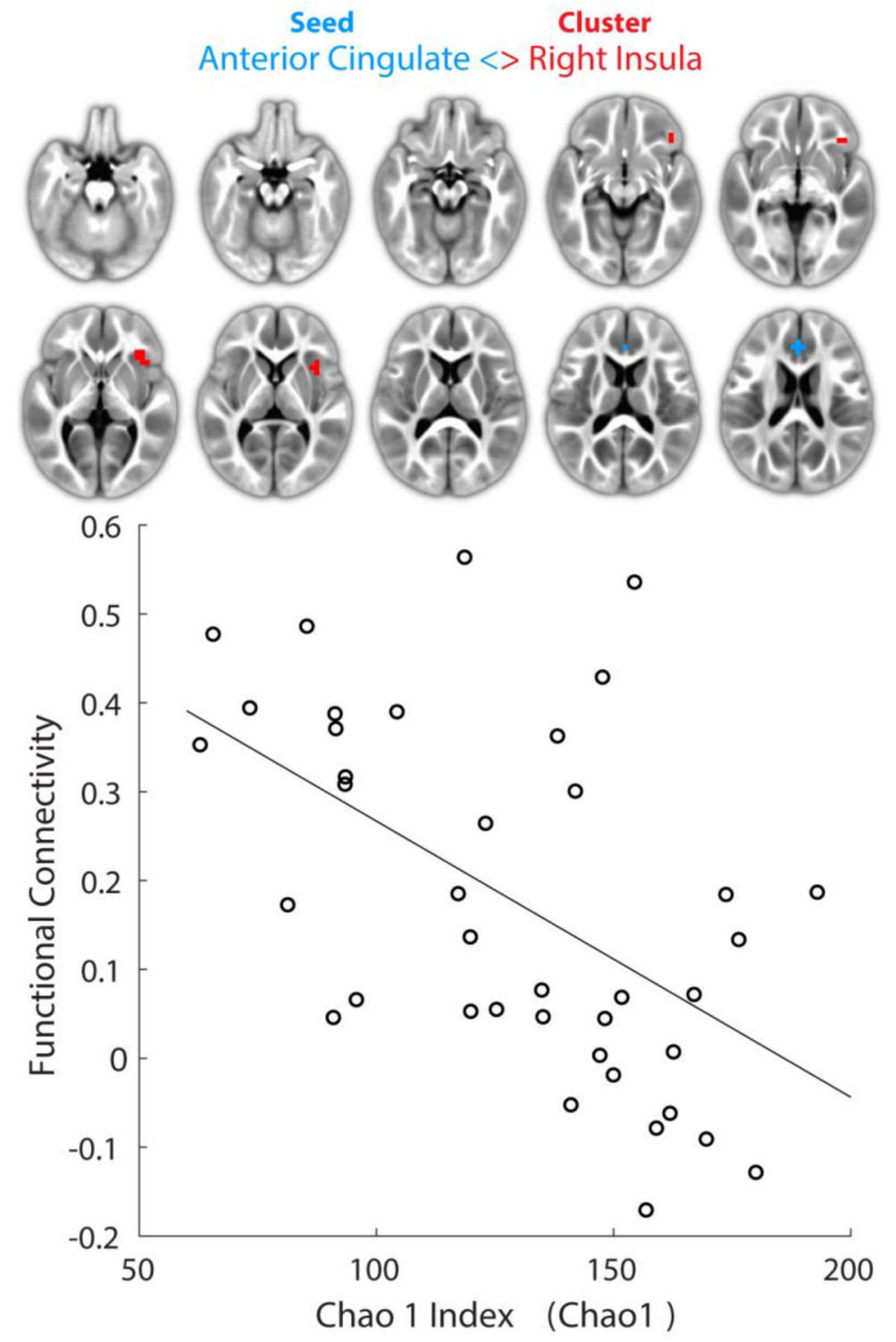

Figure 2:

Functional connectivity between the anterior cingulate cortex (seed-region, blue color) and right insula (cluster-localization) correlates with alpha diversity in one-year-olds. (a) Visualization of significant cluster $(a<0.05$; voxel-wise $\mathrm{P}<0.001$ and $7+$ face-connected voxels) located primarily within the right anterior insula. (b) Corresponding cluster-level plot of average functional connectivity versus microbiome alpha diversity (Observed Species). Data-points correspond to individual subjects $(\mathrm{N}=39)$. Line shows best linear fit; $\mathrm{r}$ $=-0.54, \mathrm{P}<0.001)$. 
a Seed Cluster

\section{Supplemental Motor Area $<>$ Inferior Parietal Lobule}
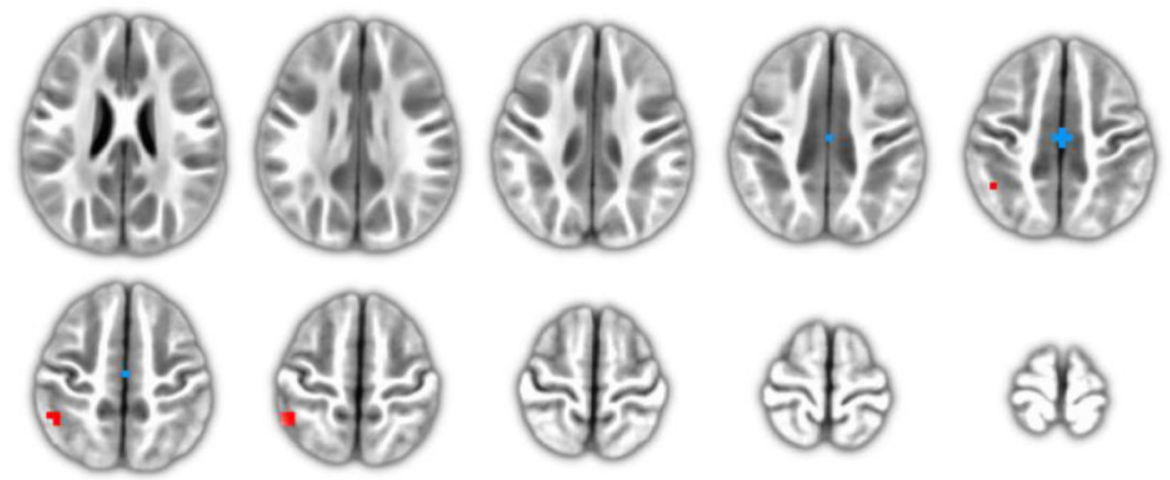

b

$$
\text { C }
$$
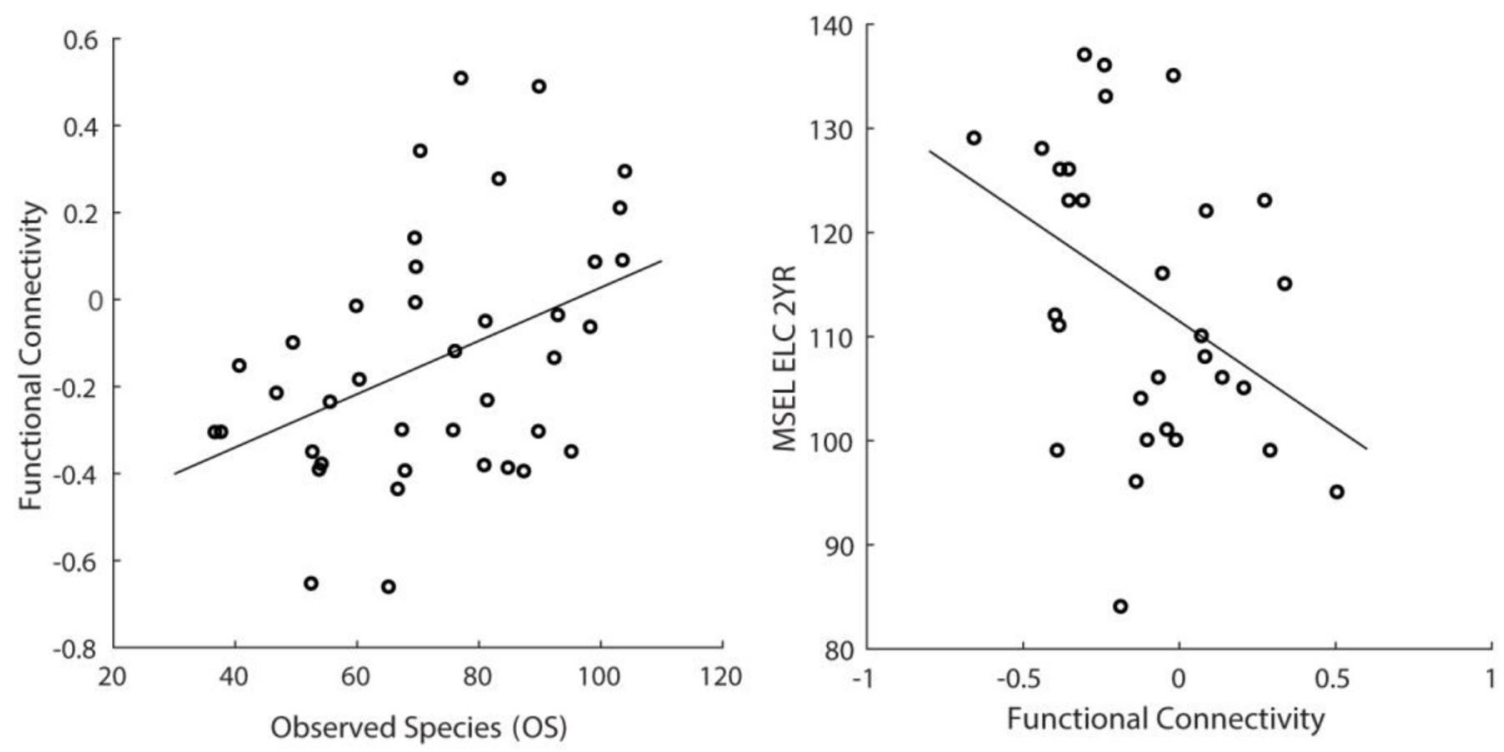

Figure 3:

Functional connectivity between the supplemental motor area (seed-region, blue color) and inferior parietal lobule (cluster-localization) correlates with alpha diversity and behavior at 2-years. (a) Visualization of significant cluster ( $a<0.05$; voxel-wise $\mathrm{P}<0.001$ and $7+$ faceconnected voxels) located in left parietal cortex. (b) Corresponding cluster-level plot of average functional connectivity versus microbiome alpha diversity (Chao 1 Index). Datapoints correspond to individual subjects $(\mathrm{N}=39)$. Line shows best linear fit; $\mathrm{r}=-0.41, \mathrm{P}=$ 0.000). (c) Corresponding cluster-level plot of 2-year behavior [Mullen Scales of Early Learning (MSEL) Early Learning Composite (ELC)] versus average functional connectivity. Data-points corresponding to individual subjects $(\mathrm{N}=30)$. Line shows best linear fit; $\mathrm{r}=$ $-0.42, \mathrm{P}=0.049$. 
Table 1:

Participant Characteristics

\begin{tabular}{|l|l|l|l|}
\hline \multirow{2}{*}{ Descriptive Variable } & & \multicolumn{2}{|l|}{ Overall } \\
\cline { 3 - 4 } & & N & $\%$ \\
\hline \multirow{3}{*}{ Income } & High & 16 & 41 \\
& Middle & 9 & 23.1 \\
& Low & 12 & 30.8 \\
& Not Available & 2 & 5.1 \\
\hline \multirow{2}{*}{ Maternal Psychiatric History } & Yes & 13 & 33.3 \\
& No & 26 & 66.7 \\
\hline \multirow{2}{*}{ Paternal Psychiatric History } & Yes & 5 & 12.8 \\
& No & 34 & 87.2 \\
\hline \multirow{2}{*}{ Cesarean Section } & Yes & 20 & 51.3 \\
& No & 19 & 48.7 \\
\hline \multirow{2}{*}{ Single or Twin Gestation } & Twin & 24 & 61.5 \\
& Singleton & 15 & 38.5 \\
\hline \multirow{2}{*}{ Sex } & Male & 16 & 41 \\
& Female & 23 & 59 \\
\hline \multirow{2}{*}{ NICU } & Yes & 10 & 25.6 \\
& No & 29 & 74.4 \\
\hline \multirow{3}{*}{ Maternal Ethnicity } & White & 32 & 82.1 \\
& Black & 6 & 15.4 \\
& Asian & 0 & 0 \\
& Native American & 1 & 2.6 \\
\hline \multirow{2}{*}{ Paternal Ethnicity } & White & 28 & 71.8 \\
& Black & 9 & 23.1 \\
& Asian & 2 & 5.1 \\
& Native American & 0 & 0 \\
\hline & & & \\
\hline
\end{tabular}

\begin{tabular}{|l|l|l|l|}
\hline \multirow{2}{*}{ Descriptive Variable } & & \multicolumn{2}{|l|}{ Overall } \\
\cline { 3 - 5 } & & N & $\%$ \\
\hline \multirow{2}{*}{ Older Siblings } & Yes & 17 & 43.6 \\
& No & 22 & 56.4 \\
\hline \multirow{2}{*}{ Currently Breastfed } & Yes & 14 & 35.9 \\
& No & 25 & 64.1 \\
\hline \multirow{2}{*}{ Ever Given Formula } & Yes & 26 & 66.7 \\
& No & 13 & 33.3 \\
\hline \multirow{2}{*}{ Currently Given Formula } & Yes & 10 & 25.6 \\
& No & 29 & 74.4 \\
\hline \multirow{2}{*}{ Given Milk Other Than Breastmilk or Formula } & Yes & 34 & 87.2 \\
& No & 5 & 12.8 \\
\hline \multirow{2}{*}{ Type of Other Milk } & Breastmilk Exclusively & 5 & 12.8 \\
& Cow's Milk & 31 & 79.5 \\
\hline \multirow{2}{*}{ Symptoms of Illness in Previous Week } & Other (almond, soy) & 3 & 7.7 \\
\hline \multirow{2}{*}{ Gastrointestinal Symptoms in Previous Week } & Yes & 21 & 53.8 \\
& No & 18 & 46.2 \\
\hline \multirow{2}{*}{ Antibiotics within Last Year } & No & 5 & 12.8 \\
\hline \multirow{2}{*}{ Antibiotics During Pregnancy } & Yes & 34 & 87.2 \\
\hline & No & 0 & 0 \\
& Yes & 39 & 100 \\
\hline
\end{tabular}




\begin{tabular}{|l|l|l|}
\hline \multirow{2}{*}{ Descriptive Variable } & \multicolumn{2}{l|}{ Overall } \\
\cline { 2 - 3 } & N & Mean \pm SD \\
\hline Gestational Age at Birth (days) & 39 & $259.5 \pm 19.5$ \\
\hline Birth Weight (grams) & 39 & $2810 \pm 716.8$ \\
\hline APGAR at 5 Minutes & 39 & $8.5 \pm 1.1$ \\
\hline Maternal Age at Birth (years) & 39 & $30.8 \pm 4.7$ \\
\hline Maternal Education (years) & 39 & $16.9 \pm 2.6$ \\
\hline Paternal Age at Birth (years) & 39 & $33 \pm 5.5$ \\
\hline Paternal Education (years) & 39 & $16 \pm 3.5$ \\
\hline Age at MRI (days) & 39 & $404.1 \pm 38.3$ \\
\hline
\end{tabular}

